\title{
REBOA for Inter-Hospital Transfer: Are We Walking in the Dark?
}

\author{
Maya Paran ${ }^{1}$, Mickey Dudkiewicz ${ }^{2}$ and Boris Kessel ${ }^{1}$ \\ 'Division of General Surgery, The Hillel Yaffe Medical Center, Hadera, affliated with The Rappaport School of Medicine, \\ Technion, Haifa, Israel \\ ${ }^{2}$ Hospital Administration, Hillel Yaffe Medical Center, Hadera, Israel
}

Worldwide, interest in resuscitative endovascular balloon occlusion of the aorta (REBOA) for different indications is consistently growing. Initially implemented for trauma and vascular surgery, the current literature includes multiple publications reflecting successful broadening indications for the use of REBOA, including non-traumatic bleeding, obstetrics catastrophes, prehospital care, and many others. Over recent years, experience with REBOA has been growing with a better understanding of various physiological processes occurring during REBOA use. Continuous efforts are being made to increase and diversify REBOA indications. Recently, we are witnessing an attempt to insert REBOA for inter-hospital transfer.

Transfer of patients to a higher level-of-care hospital is, without a doubt, an important aspect of trauma care, aimed to provide optimal care for all trauma patients. The key fundamentals of this sophisticated process involve the decision to transfer, strict protocols of communication between hospitals, choosing the optimal transportation mode and the appropriate team, and ensuring adequate documentation of events occurring before and during the transfer. The central and probably the most important element is the decision regarding pretransfer patient's stabilization procedures. The question of whether unstable patients may be transported safely remains open and has been readdressed in multiple debates.

May REBOA make trauma patients' transfer safer? There are multiple reports of successful "out-of-hospital" REBOA use, including military/other austere environment settings as well as civil prehospital REBOA [1-4]. Lastly, several reports of REBOA performed for

\section{Corresponding author:}

Maya Paran, MD, Hillel Yaffe Medical Center, Ha-Shalom St., Hadera, 3846201, Israel.

Email: paran.maya@gmail.com

(C) 2021 CC BY 4.0 - in cooperation with Depts. of Cardiothoracic/ Vascular Surgery, General Surgery and Anesthesia, Örebro University Hospital and Örebro University, Sweden inter-hospital transfer have been published, including in the pages of JEVTM. For example, Beldowicz et al., in the first REBOA use for inter-hospital transfer, described a balloon inflation time of nearly two hours when previously unprepared paramedics were instructed to inject an additional $4 \mathrm{~mL}$ of saline into the balloon whenever the patient became hypotensive during the transport [5]. The possible consequences of REBOA use during transportation have been well described in a review study by Goforth et al. [6]. However, even when reporting on a safe air evacuation, the authors concluded that, due to the risk of potentially life-threatening balloon migration, REBOA is contraindicated during flight [6]. Nevertheless, it remains unclear which data supports this conclusion.

In our certainly debatable opinion, these publications raise several concerns that should be evaluated and addressed before any conclusions can be made. First, we assume that significant differences exist between prehospital or military settings, where REBOA serves as a last resort to get the patient alive to reasonable medical care and inter-hospital transfer. We are completely aware of the variety of different national trauma systems, times of transportation, and distances between countries. The reported American College of Surgeons (ACS) definitions of trauma care levels also vary significantly between countries. For example, in Israel, the single difference between a Level I and II trauma center is a lack of cardiothoracic and neurosurgical services in a Level II center. Therefore, REBOA use for inter-hospital transfer, in the vast majority of cases, is not relevant in most situations where a patient is being transferred from a Level II to a Level I center. The reality is very different in larger countries, with longer transportation times and greater differences in the abilities of various trauma centers.

Adaptation of REBOA use for inter-hospital transfer requires addressing some fundamental questions. When the patient is stable before the transfer, is there any role for insertion of a REBOA balloon without inflation? Is it to reassure the referring team only? Or is it rather a new tool that may mark a new transfer era and allow for a safer transfer? Do we really know how many initially 
stable/resuscitation-responding patients have deteriorated during their transportation?

In this editorial, we call for action and for the conduction of a multicenter study, which may shed some light on this issue. When the patient is unstable before the transfer, is there any role for the REBOA balloon inside? After all, we know that the maximal safe inflation time for REBOA is approximately 60 minutes. How should we act in cases when the expected transportation time is much longer? Is the use of partial/intermittent REBOA during the transport safe? Especially when performed by an inexperienced team? Perhaps better definitions of specific scenarios in which a patient should be evacuated directly to a Level I trauma center could prevent the need for inter-hospital transfer.

In summary, we believe that there are more questions than answers regarding this matter. In order to shed some light on this topic, we would like to emphasize the need for an open discussion in this journal.

\section{REFERENCES}

[1] Northern DM, Manley JD, Lyon R, et al. Recent advances in austere combat surgery: use of aortic balloon occlusion as well as blood challenges by special operations medical forces in recent combat operations. J Trauma Acute Care Surg. 2018;85(1S Suppl 2): S98-S103.

[2] Stokes SC, Theodorou CM, Zakaluzny SA, DuBose JJ, Russo RM. Resuscitative endovascular balloon occlusion of the aorta in combat casualties: the past, present, and future. J Trauma Acute Care Surg. 2021;91(2S Suppl 2): S56-S64.

[3] Sadek S, Lockey DJ, Lendrum RA, Perkins Z, Price J, Davies GE. Resuscitative endovascular balloon occlusion of the aorta (REBOA) in the pre-hospital setting: an additional resuscitation option for uncontrolled catastrophic haemorrhage. Resuscitation. 2016;107:135-8.

[4] Lamhaut L, Qasim Z, Hutin A, et al. First description of successful use of zone 1 resuscitative endovascular balloon occlusion of the aorta in the prehospital setting. Resuscitation. 2018;133:e1-e2.

[5] Beldowicz B, Salcedo E, Galante J. Resuscitative endovascular balloon occlusion of the aorta in inter-hospital transfers: two case reports. J Endovasc Resusc Trauma Manag. 2018;2(1):44-8.

[6] Goforth C, Bradley M, Pineda B, See S, Pasley J. Resuscitative endovascular balloon occlusion of the aorta: a bridge to flight survival. Crit Care Nurse. 2018;38(2): 69-75. 